\title{
Distribution of avian influenza viruses according to environmental surveillance during 2014-2018, China
}

Hong Bo ${ }^{1}$, Ye Zhang ${ }^{1}$, Li-Bo Dong ${ }^{1}$, Jie Dong ${ }^{1}$, Xi-Yan Li ${ }^{1}$, Xiang Zhao ${ }^{1}$, Zi Li ${ }^{1}$, Yue-Long Shu ${ }^{2}$ and Da-Yan Wang ${ }^{\text {** }}$

\begin{abstract}
Background: Recurrent infections of animal hosts with avian influenza viruses (AIVs) have posted a persistent threat. It is very important to understand the avian influenza virus distribution and characteristics in environment associated with poultry and wild bird. The aim of this study was to analyze the geographic and seasonal distributions of AIVs in the 31 provinces, municipalities and autonomous region (PMA) of China, compare the Alvs prevalence in different collecting sites and sampling types, analyze the diversity of AIVs subtypes in environment.

Methods: A total of 742005 environmental samples were collected from environmental samples related to poultry and wild birds in different locations in the mainland of China during 2014-2018. Viral RNA was extracted from the environmental samples. Real-time RT-PCR assays for influenza $\mathrm{A}, \mathrm{H} 5, \mathrm{H} 7$ and $\mathrm{H} 9$ subtypes were performed on all the samples to identify subtypes of influenza virus. The nucleic acid of influenza A-positive samples were inoculated into embryonated chicken eggs for virus isolation. Whole-genome sequencing was then performed on Illumina platform. SPSS software was used to paired t test for the statistical analysis. ArcGIS was used for drawing map. Graphpad Prism was used to make graph.
\end{abstract}

Results: The nucleic acid positivity rate of influenza $\mathrm{A}, \mathrm{H5}, \mathrm{H} 7$ and $\mathrm{H} 9$ subtypes displayed the different characteristics of geographic distribution. The nucleic acid positivity rates of influenza A were particularly high $(25.96 \%-45.51 \%)$ in eleven provinces covered the Central, Eastern, Southern, Southwest and Northwest of China. The nucleic acid positivity rates of $\mathrm{H} 5$ were significantly high (11.42\%-13.79\%) in two provinces and one municipality covered the Southwest and Central of China. The nucleic acid positivity rates of $\mathrm{H} 7$ were up to $4 \%$ in five provinces covered the Eastern and Central of China. The nucleic acid positivity rates of $\mathrm{H} 9$ were higher (13.07\%-2.07\%) in eleven PMA covered the Southern, Eastern, Central, Southwest and Northwest of China. The nucleic acid positivity rate of influenza A, H5, H7 and $\mathrm{H} 9$ showed the same seasonality. The highest nucleic acid positivity rates of influenza A, H5, H7, H9 subtypes were detected in December and January and lowest from May to September. Significant higher nucleic acid positivity rate of influenza $\mathrm{A}, \mathrm{H} 5, \mathrm{H} 7$ and $\mathrm{H} 9$ were detected in samples collected from live poultry markets (LPM) $(30.42 \%, 5.59 \%$, $4.26 \%, 17.78 \%)$ and poultry slaughterhouses $(22.96 \%, 4.2 \%, 2.08 \%, 12.63 \%)$. Environmental samples that were collected from sewage and chopping boards had significantly higher nucleic acid positivity rates for influenza A (36.58\% and $33.1 \%), \mathrm{H} 5$ (10.22\% and 7.29\%), H7(4.24\% and 5.69\%)and $\mathrm{H} 9(21.62 \%$ and $18.75 \%)$. Multiple subtypes of Alvs

\footnotetext{
*Correspondence: wangdayan@ivdc.chinacdc.cn

${ }^{1}$ National Institute for Viral Disease Control and Prevention, Chinese Center for Disease Control and Prevention; WHO Collaborating Center for Reference and Research On Influenza; Key Laboratory for Biosafety, National Health Commission, Beijing, China

Full list of author information is available at the end of the article
} permits use, sharing, adaptation, distribution and reproduction in any medium or format, as long as you give appropriate credit to the original author(s) and the source, provide a link to the Creative Commons licence, and indicate if changes were made. The images or other third party material in this article are included in the article's Creative Commons licence, unless indicated otherwise in a credit line to the material. If material is not included in the article's Creative Commons licence and your intended use is not permitted by statutory regulation or exceeds the permitted use, you will need to obtain permission directly from the copyright holder. To view a copy of this licence, visit http://creativecommons.org/licenses/by/4.0/. The Creative Commons Public Domain Dedication waiver (http://creativeco mmons.org/publicdomain/zero/1.0/) applies to the data made available in this article, unless otherwise stated in a credit line to the data. 
including nine hemagglutinin (HA) and seven neuraminidase (NA) subtypes were isolated form the environmental samples. The $\mathrm{H} 5, \mathrm{H} 7$, and $\mathrm{H} 9$ subtypes accounted for the majority of AlVs in environment.

Conclusions: In this study, we found the avian influenza viruses characteristics of geographic distribution, seasonality, location, samples types, proved that multiple subtypes of AIVs continuously coexisted in the environment associated with poultry and wild bird, highlighted the need for environmental surveillance in China.

Keywords: Avian influenza virus, Live poultry market, Environment, Surveillance

\section{Background}

AIVs were first reported in 1878 [1] in Italy and were subsequently isolated from chickens in 1934 [2]. AIVs are categorized into two pathotypes according to their virulence in chickens: low-pathogenic and highly pathogenic AIVs [3, 4]. Of the 16 HA subtypes that have been identified in birds, 5 HA subtypes of AIVs (H5N1, H5N6, H5N8, H6N1, H7N9, H7N2, H7N3, H7N7, H9N2, H10N7, and H10N8) are known to cause human infections $[5,6]$, among which the H5 subtype has a nearly global distribution in birds. Moreover, the H7N9 subtype widely circulates and had rapidly evolved in LPMs in China especially from 2013 to 2018 [7, 8]. Furthermore, continuous reassortments among AIV strains have increased the risk of a pandemic.

Many studies have investigated the demographic and ecological risk factors associated with the effective transmission of AIVs. LPMs play an important role in human infection with AIVs. Bird transport between LPMs affected the emergence of H7N9 in Eastern China, and the closure of LPMs reduced the incidence of human infection with AIVs [9]. Furthermore, one survey found that $80 \%$ of households that purchased poultry from LPMs had an increased risk of poultry-to-human infection [10]. LPMs are particularly common in Southern China, and many subtypes, such as H9, H5, and H6, have been enzootic in poultry in China since the mid-1990s [11]. However, environmental factors of LPM, such as temperature, humidity, and feeding conditions, play important roles in AIV survival and infectivity [12].

Environmental surveillance related poultry and wild bird was established in China in 2009. Samples are collected each month by each province. In the present study, we analyzed the environmental samples collected from 2014 to 2018 as part of this programme to examine the geographic and seasonal distributions of AIVs in China and how these vary with regard to sites and sample types to determine environmental risk factors.

\section{Methods}

\section{Environmental sample collection sites}

Samples were collected from 2014 to 2018 by Chinese National Influenza Surveillance Network laboratories. Based on the national surveillance guidelines, at least
40 samples per month were collected from each of the 31 PAM in China located in seven regions (Eastern, Southern, Central, Northern, Northwest, Southwest, and Northeast of China), providing a total of 742,005 samples. These samples were obtained from a range of sites, including LPMs, poultry farms, backyards, slaughterhouses and wild bird habitats. These samples were obtained from a variety of poultry-related materials, including poultry faeces, drinking water, sewage, and swabs from poultry cages, feathers, etc. Each sample was maintained in viral transport medium and immediately transported at a low temperature to the nearest Chinese National Influenza Surveillance Network laboratory.

\section{Environmental sample collection}

Faeces or swab samples were put into $5 \mathrm{ml}$ Hank's medium containing $0.5 \%$ bovine albumin, ampicillin $\left(2 \times 10^{6} \mathrm{IU} / \mathrm{L}\right)$, streptomycin $(200 \mathrm{mg} / \mathrm{L})$, polymyxin B $\left(2 \times 10^{6} \mathrm{IU} / \mathrm{L}\right)$, gentamicin $(250 \mathrm{mg} / \mathrm{L})$, mycin $\left(0.5 \times 10^{6} \mathrm{IU} / \mathrm{L}\right)$, oxygen hydrochloride floxacin $(60 \mathrm{mg} / \mathrm{L})$, and sulfamethoxazole $(200 \mathrm{mg} / \mathrm{L})$. For liquid samples (drinking water, sewage), 5-ml liquid was collected. The samples were sent to a local network laboratory within $48 \mathrm{~h}$ and stored at $4{ }^{\circ} \mathrm{C}$. The samples were mixed thoroughly, centrifuged at $3000 \mathrm{rpm}$ for $10 \mathrm{~min}$, and the supernatants were aliquoted into three tubes. Each tube contained about $1.5 \mathrm{ml}$ of the sample; one was designated for nucleic acid identification by a local network laboratory, one was designated for virus isolation, and one was transported to the Chinese National Influenza Center and then stored at $-80{ }^{\circ} \mathrm{C}$ for further test.

\section{RNA extraction}

Viral RNA was extracted from each of the collected samples using a QIAsymphony RNA Kit (931636; Qiagen, Hilden, Germany) with a QIAsymphony SP instrument (Qiagen) according to the manufacturer's instructions.

\section{Real-time RT-PCR}

Real-time RT-PCR assays for influenza A, H5, H7, and H9 subtypes were performed on all the samples, with primer and probe sets provided in the Chinese National Influenza Surveillance Guidelines (Additional file 1: Table S1). The reactions were carried out using an AgPath-ID ${ }^{\mathrm{TM}}$ 
One-Step RT-PCR Kit (4387422; Ambion ${ }^{\circledR}$ ) under the following cycling conditions: $10 \mathrm{~min}$ at $45{ }^{\circ} \mathrm{C} ; 10 \mathrm{~min}$ at $95{ }^{\circ} \mathrm{C} ; 40$ cycles of $15 \mathrm{~s}$ at $95{ }^{\circ} \mathrm{C}$; and $45 \mathrm{~s}$ at $60{ }^{\circ} \mathrm{C}$. The positive control contained all the reaction components and RNA of influenza A, and H5, H7, and $\mathrm{H} 9$ subtypes. The negative control contained all the reaction components and $\mathrm{H}_{2} \mathrm{O}$ as the template.

\section{Virus isolation}

Virus isolation was performed on the influenza A-positive samples detected by Real-time RT-PCR. The samples were inoculated into the allantoic cavity of 9 to 10-dayold embryonated chicken eggs; the eggs were incubated at $37{ }^{\circ} \mathrm{C}$ for $48 \mathrm{~h}$ and chilled at $4{ }^{\circ} \mathrm{C}$ overnight. The allantoic fluid was then harvested, and a haemagglutination assay was performed using $1 \%$ turkey red blood cells to determine the titer of harvested viruses [13].

\section{Next-generation sequencing}

Virus total RNA was extracted by a MagMAX ${ }^{\mathrm{TM}}$ Viral/ Pathogen Nucleic Acid Isolation Kit (cat\# 42352, Applied Biosystems). The RNA was subjected to reverse transcription and amplification using the SuperScript ${ }^{\text {TM }}$ III One-Step RT-PCR System with Platinum ${ }^{\text {TM }}$ Taq High Fidelity DNA Polymerase (cat\#: 12574035, Invitrogen). The DNA was purified by a MagMax Core Nucleic Acid Purification Kit (cat\# 1903031, Thermo Fisher Scientific). The DNA library was prepared using Nextera XT DNA Preparation Kits (cat\#FC-131-1096, Illumina). Wholegenome sequencing was then performed on MiSeq highthroughput sequencing platform and Nextseq platform (Illumina, Inc., San Diego, CA, USA), and the data were analysed using CLC Genomics Workbench software.

\section{Statistical analysis}

The statistical software SPSS version 25 (IBM Corp, New York, USA) was used to data statistical analysis. A paired $t$ test was performed to analyze the significant difference. $P<0.05$ was considered significant.

\section{Map plotting}

ArcGIS version 10.7 (Environmental Systems Research Institute, RedLands, USA) was used to draw the map. Different colors represented the range of positivity rate on the map.

\section{Graph making}

Graphpad Prism5 (Graphpad software Inc, San Diego, USA) was used to process the data and made graph.

\section{Results}

Geographic distributions of avian influenza viruses

Based on RT-PCR, the positivity rate for influenza A was $22.57 \%$ (Fig. 1a). The positivity rates were particularly high $(25.96 \%-45.51 \%)$ in 11 provinces in five of the seven regions tested: Central China (Hunan provinces), Eastern China (Fujian, Jiangxi and Zhejiang provinces), Southern China (Guangxi Autonomous Region), Southwest China (Chongqing Municipality and Guizhou Province), and Northwest China (Gansu Province).

Among the influenza A viruses detected, the H5 subtype was detected in $3.89 \%$ of the samples (Fig. 1b). The positivity rates were significantly high $(11.42 \%-13.79 \%)$ in two provinces and one municipality in two regions: Southwest China (Chongqing Municipality), Central China (Hunan and Jiangxi provinces).

The positivity rate for the $\mathrm{H} 7$ subtype was $3.41 \%$ (Fig. 1c), with rates of up to $4 \%$ being detected in two regions: Eastern China (Jiangsu, Fujian, Zhejiang and Jiangxi provinces) and Central China (Hunan Province).

The positivity rate for the H9 subtype is 13.07\% (Fig. 1d), with 11 PAM displayed high positivity rates (13.07\%-27.07\%). These regions included Southern China (Guangdong Province and Guangxi Autonomous Region), Eastern China (Zhejiang, Anhui, Jiangxi and Fujian provinces), Central China (Hunan Province), Southwest China (Yunnan Province and Chongqing Municipality) and Northwest China (Gansu Province and Xinjiang Autonomous Region). The nucleic acid positivity rates of influenza A H5, H7, and H9 subtypes in different regions were shown in Fig. 1 and Additional file 1: Table S2.

\section{Seasonality of avian influenza viruses in environments}

The monthly nucleic acid positivity rates of influenza A, H5, H7 and $\mathrm{H} 9$ subtypes in the poultry-related environmental samples were shown in Fig. 2 and Additional file 1: Table S3. The positivity rates of influenza A, H5, H7 and $\mathrm{H} 9$ subtypes showed obvious seasonality and were highest in December and January and lowest from May to September.

\footnotetext{
Variations of positivity rates of influenza $\mathrm{A}, \mathrm{H} 5, \mathrm{H} 7$ and $\mathrm{H} 9$ subtypes among the sampling sites

During 2014-2018, samples with the highest positivity rates of influenza $\mathrm{A}, \mathrm{H} 5, \mathrm{H} 7$ and $\mathrm{H} 9$ were collected from LPMs (30.42\%, 5.59\%, 4.26\%, 17.78\%), followed by slaughterhouses $(22.96 \%, 4.2 \%, 2.08 \%, 12.63 \%)$, respectively. In
} 


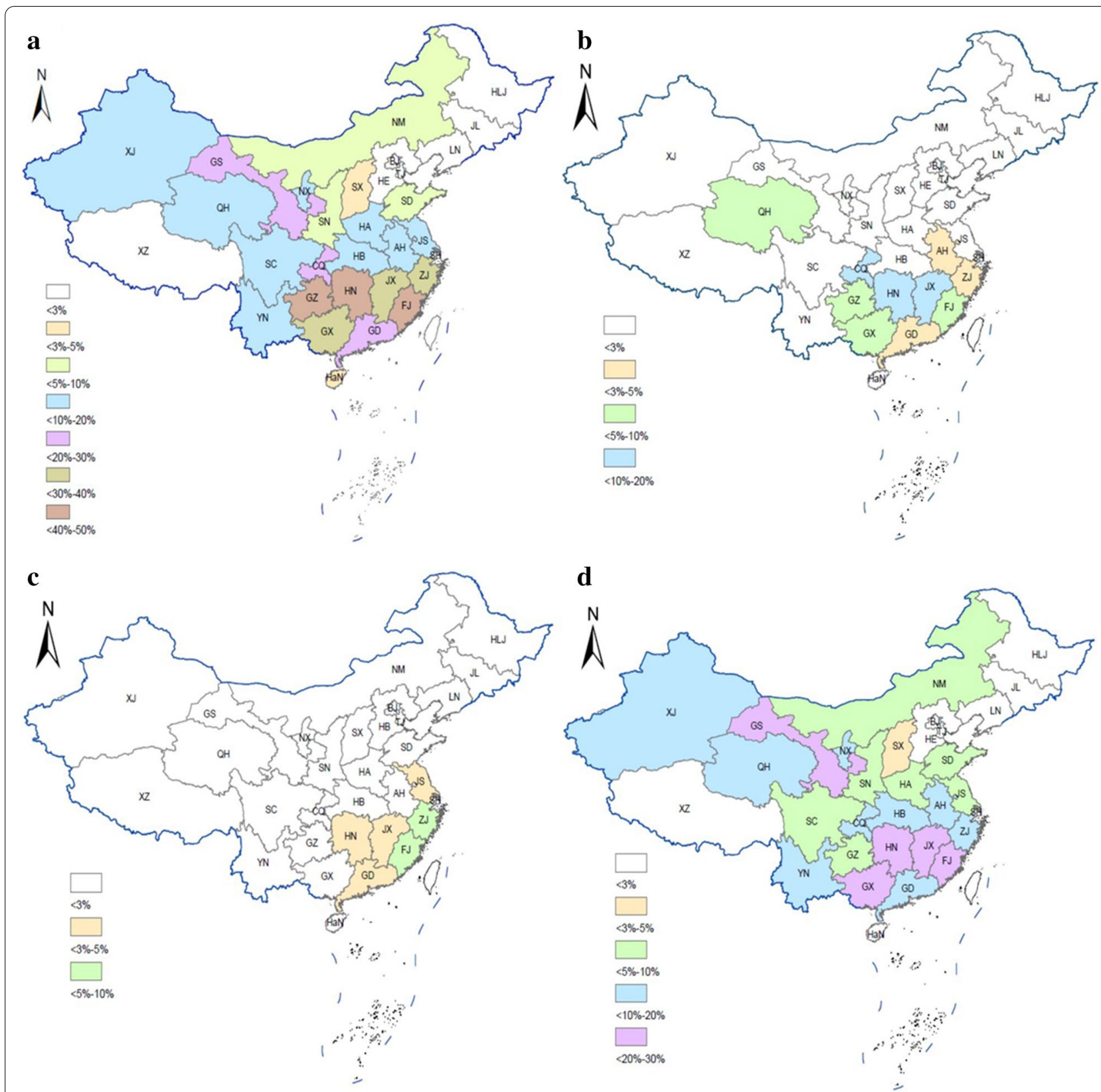

Fig. 1 Geographical distributions of the nucleic acid positivity rates of influenza A, H5, H7 and $\mathrm{H} 9$ subtypes. a-d indicates the nucleic acid positivity rates of influenza A, H5, H7 and H9 subtypes on average in 31 PMA during 2014-2018, respectively. Different colours represent the corresponding range of positivity rates. Abbreviations: AH, Anhui; BJ, Beijing; CQ, Chongqing; FJ, Fujian; GD, Guangdong; GS, Gansu; GX, Guangxi; GZ, Guizhou; HA, Henan; HB, Hubei; HE, Hebei; HL, Heilongjiang; HN, Hunan; HaN, Hainan; JL, Jilin; JS, Jiangsu; JX, Jiangxi; LN, Liaoning; NM, Inner Mongolia; NX, Ningxia; QH, Qinghai; SC, Sichuan; SD, Shandong; SH, Shanghai; SX, Shanxi; SN, Shaanxi; TJ, Tianjin; XJ, Xinjiang; XZ, Tibet; YN, Yunnan; ZJ, Zhejiang. PAM: Provinces, municipalities and autonomous regions

contrast, poultry farms, backyards and wild bird habitats had influenza A positivity rates of $3.26 \%, 3.44 \%$, and $1.1 \%$ respectively, while the $\mathrm{H} 5$ and $\mathrm{H} 7$ positivity rates were all less than $1 \%$. Poultry farms, backyards, and wild bird habitats had $\mathrm{H} 9$ positivity rates of $1.98 \%, 1.42 \%$, and
$0.25 \%$. The statistical analysis indicated that LPMs and slaughterhouses were associated with significantly higher positivity rates of AIVs than all other sites during the study period $(P<0.05$; Table 1$)$. 
Variations of the positivity rates of influenza $A, \mathrm{H} 5, \mathrm{H} 7$ and $\mathrm{H} 9$ subtypes among different sample types

Environmental samples that were collected from sewage and chopping boards had significantly higher positivity rates for influenza A (36.58\% and 33.1\%), H5 (10.22\% and $7.29 \%), \mathrm{H} 7$ (4.24\% and 5.69\%) and H9 (21.62\% and $18.75 \%)$ than those collected from faeces, cages, and feeding troughs $(P<0.05)$. Furthermore, the positivity rates of $\mathrm{H} 9$ in samples originating from sewage and chopping boards were significantly higher than those of $\mathrm{H} 5$ and $\mathrm{H} 7$ subtypes $(P<0.05$; Table 2$)$.

\section{Multiple subtypes of influenza A viruses were detected in poultry-related environments}

In total, nine HA subtypes and seven NA subtypes of avian influenza viruses were isolated including the HA subtypes H1, H3, H4, H5, H6, H7, H9, H10, and H11, and the NA subtypes N1, N2, N3, N6, N7, N8, and N9. In general, the $\mathrm{H} 5, \mathrm{H} 7$, and $\mathrm{H} 9$ subtypes of influenza A virus accounted for the majority of avian influenza viruses isolated from the environmental samples (Additional file 1: Table S4). The proportion of H9N2 avian influenza viruses took a proportion of $46.90 \%$ among all the subtypes. The proportion of $\mathrm{H} 5 \mathrm{~N} 6$ and $\mathrm{H} 5 \mathrm{~N} 1 \mathrm{AIVs}$ were $20.66 \%$ and $6.31 \%$. The proportion of H7N9 AIVs was $8.9 \%$. The proportion of other subtypes were less than $0.5 \%$ except for H3N2 (6.15\%), H6N6 (4.43\%), and mixture subtypes (1.58\%). The H9N2, H5N6 and H7N9 AIVs displayed obviously different proportions from 2014 to 2018. The proportion of subtype H5N6 AIVs increased more than threefold from $11 \%$ in 2014 to $34 \%$ in 2016 , then decreased to $12.14 \%$ in 2017. The H5N1 AIVs showed a declining trend from 2014 to 2018 . The proportion of H7N9 AIVs reached 23\% in 2017, which was approximately four times that in 2014 (6\%) and decreased to $0.26 \%$ in 2018 . The proportion of H9N2 AIVs showed a decreasing from 2014 to 2017 , with proportions of $59 \%$, $51 \%, 35 \%$ and $36 \%$, respectively then increased to $53.36 \%$ in 2018 (Fig. 3 and Additional file 1: Table S4).

\section{Discussion}

Based on five-year environmental surveillance data, we analysed the geographical distributions of influenza A, $\mathrm{H} 5, \mathrm{H} 9$ and $\mathrm{H} 7$ subtypes in AIVs related environment. The geographical distribution of $\mathrm{H} 7$ subtype mainly occurred in the Yangtze and Pearl River deltas and a few adjacent provinces. It was reported that these regions were the sources of newly emerged H7N9 human infections during 2013-2017 [11]. Poultry is the major source of H7N9 infections in humans [14]. Our study indicates that the $\mathrm{H} 7$ subtype persists in the environment in these regions and may be associated with human infection and virus evolution. The geographical distribution of H5 was mainly located in Southern China. H5 has been widely circulating among poultry in China since 2004. The highly pathogenic avian influenza H5 subtype has evolved multiple clades and subclades [15]. Though control measures were carried out, LPMs and other places are still providing the opportunity for transmission and evolution of $\mathrm{H} 5$ subtype, birds and poultry still carry and transmit the H5 AIVs. Compared with the $\mathrm{H} 5$ and H7 subtypes, the H9 subtype geographic distribution was nationwide, and it was especially prevalent in the southern and western regions of China. The first H9N2

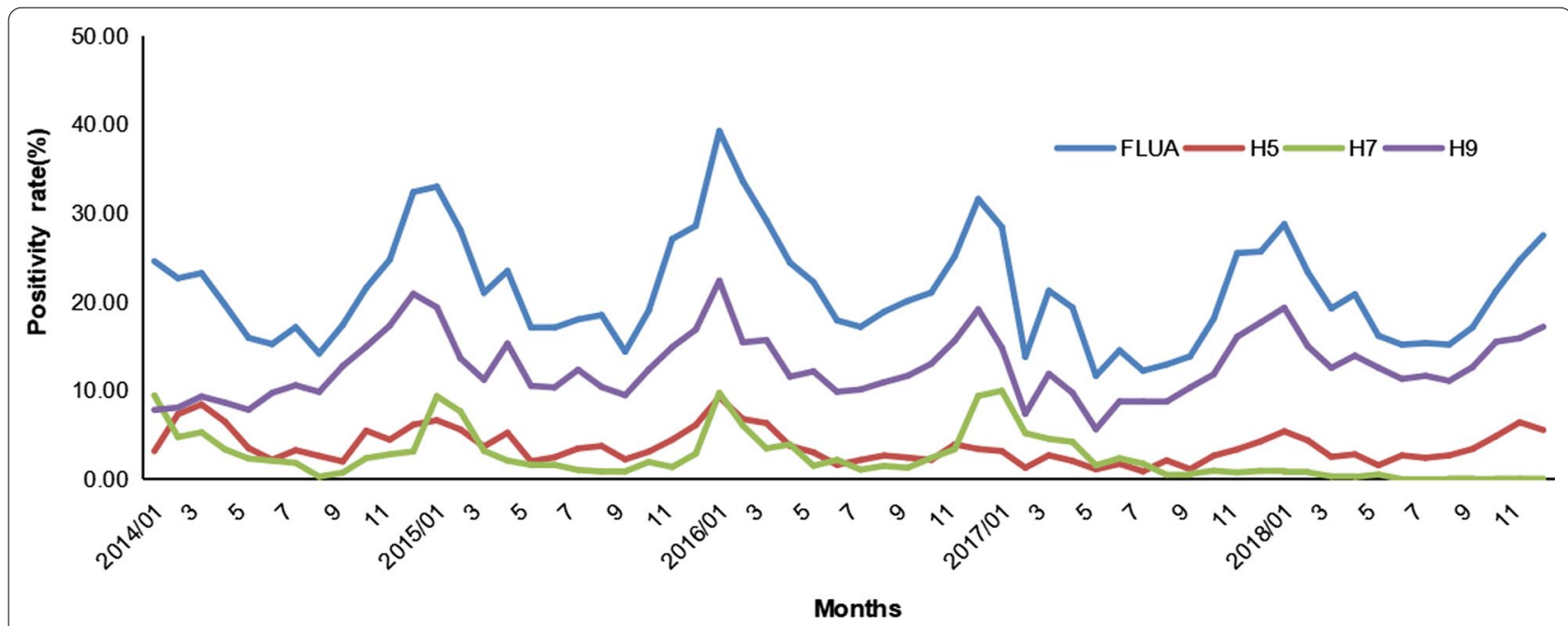

Fig. 2 Monthly nucleic acid positivity rates of influenza $\mathrm{A}, \mathrm{H} 5, \mathrm{H} 7$ and $\mathrm{H} 9$ subtypes. The blue line represents the nucleic acid positivity rate of influenza A; the red line represents the nucleic acid positivity rate of $\mathrm{H} 5$ subtype; the light green line represents the nucleic acid positivity rate of $\mathrm{H} 7$ subtype; the purple line represents the nucleic acid positivity rate of $\mathrm{H} 9$ subtype 
Table 1 Influenza A, H5, H7 and H9 nucleic acid positivity rates of different sites

\begin{tabular}{|c|c|c|c|c|c|}
\hline \multirow{3}{*}{$\begin{array}{l}\text { Type/subtype } \\
\text { Year }\end{array}$} & \multicolumn{5}{|l|}{ Positivity rate (\%) } \\
\hline & \multicolumn{5}{|c|}{ (Number of positive samples/Number of samples collected) } \\
\hline & LPM & Slaughterhouse & Backyard & Poultry farm & Wild bird habitat \\
\hline \multicolumn{6}{|l|}{ Influenza A } \\
\hline 2014 & $30.61(12050 / 39365)$ & $16.67(372 / 2232)$ & $1.69(82 / 4847)$ & $2.31(242 / 10458)$ & $4.12(86 / 2086)$ \\
\hline 2015 & $30.2(14245 / 47174)$ & $19.16(396 / 2067)$ & $4.23(185 / 4373)$ & $2.85(270 / 9470)$ & $0.53(11 / 2080)$ \\
\hline 2016 & $35.24(17910 / 50882)$ & $25.04(475 / 1897)$ & $5.4(285 / 5274)$ & 4.69(546/11649) & $0.35(7 / 2003)$ \\
\hline 2017 & $25.98(20620 / 79377)$ & $23.70(720 / 3039)$ & $2.83(341 / 12036)$ & $3.33(508 / 15251)$ & $0.33(10 / 3076)$ \\
\hline 2018 & $30.06(14428 / 47999)$ & $30.22(727 / 2406)$ & $3.04(246 / 8098)$ & $3.10(332 / 10705)$ & $0.19(5 / 2683)$ \\
\hline Mean \pm SD & $30.42 \pm 3.28$ & $22.96 \pm 5.28$ & $3.44 \pm 1.41$ & $3.26 \pm 0.88$ & $1.1 \pm 1.69$ \\
\hline \multicolumn{6}{|l|}{$\mathrm{A} / \mathrm{H} 5$} \\
\hline 2014 & $7.2(2833 / 39365)$ & $3.18(71 / 2232)$ & $0.21(10 / 4847)$ & $0.73(76 / 10458)$ & $0(0 / 2086)$ \\
\hline 2015 & $5.94(2802 / 47174)$ & $3.87(80 / 2067)$ & $0.8(225 / 4373)$ & $0.29(27 / 9470)$ & $0.1(2 / 2080)$ \\
\hline 2016 & $6.01(3057 / 50882)$ & $5.01(95 / 1897)$ & $0.36(19 / 5274)$ & $0.29(34 / 11649)$ & $0.1(2 / 2003)$ \\
\hline 2017 & $3.24(2572 / 79377)$ & $2.47(75 / 3039)$ & $0.19(23 / 12036)$ & $0.19(29 / 15251)$ & $0(0 / 3016)$ \\
\hline 2018 & $5.54(2658 / 47999)$ & $6.48(156 / 2406)$ & $0.3(24 / 8098)$ & $0.45(48 / 10705)$ & $0(0 / 2683)$ \\
\hline Mean $\pm S D$ & $5.59 \pm 1.45$ & $4.2 \pm 1.58$ & $0.37 \pm 0.24$ & $0.39 \pm 0.21$ & $0.04 \pm 0.05$ \\
\hline \multicolumn{6}{|l|}{$\mathrm{A} / \mathrm{H} 7$} \\
\hline 2014 & $5.43(2139 / 39365)$ & $0.76(17 / 2232)$ & $0.52(25 / 4847)$ & $0.71(74 / 10458)$ & $1.39(29 / 2086)$ \\
\hline 2015 & $4.73(2231 / 47174)$ & $2.18(45 / 2067)$ & $0.8(35 / 4373)$ & $0.04(4 / 9470)$ & $0(0 / 2080)$ \\
\hline 2016 & $5.57(2832 / 50882)$ & $3.22(61 / 1897)$ & $1.31(69 / 5274)$ & $0.25(29 / 11649)$ & $0.05(1 / 2003)$ \\
\hline 2017 & $5.15(4090 / 79377)$ & $2.57(78 / 3039)$ & $0.75(90 / 12036)$ & $1.36(207 / 15251)$ & $0(0 / 3016)$ \\
\hline 2018 & $0.42(200 / 47999)$ & $1.66(40 / 2406)$ & $0.14(11 / 8098)$ & $0.05(5 / 10705)$ & $0(0 / 2683)$ \\
\hline Mean $\pm S D$ & $4.26 \pm 2.17$ & $2.08 \pm 093$ & $0.7 \pm 0.42$ & $0.48 \pm 0.56$ & $0.28 \pm 0.6$ \\
\hline \multicolumn{6}{|l|}{$\mathrm{A} / \mathrm{H} 9$} \\
\hline 2014 & $15.68(6173 / 39365)$ & $8.47(189 / 2232)$ & $0.8(39 / 4847)$ & $1.1(115 / 10458)$ & $0.48(10 / 2086)$ \\
\hline 2015 & $18.04(8509 / 47,174)$ & $9.82(203 / 2067)$ & $2.74(120 / 4373)$ & $1.19(113 / 9470)$ & $0.24(5 / 2080)$ \\
\hline 2016 & $19.51(9915 / 5088)$ & $13.86(263 / 1897)$ & $3.3(174 / 5274)$ & $1.48(172 / 11649)$ & $0.2(4 / 2003)$ \\
\hline 2017 & $15.05(11950 / 79377)$ & $14.61(444 / 3039)$ & $1.37(165 / 12036)$ & $1.24(189 / 15251)$ & $0.2(6 / 3016)$ \\
\hline 2018 & 20.61 (9893/47999) & $16.38(394 / 2406)$ & $1.68(136 / 8098)$ & $2.10(225 / 10705)$ & $0.112(3 / 2683)$ \\
\hline Mean \pm SD & $17.78 \pm 2.39$ & $12.63 \pm 3.34$ & $1.98 \pm 1.02$ & $1 . .42 \pm 0.4$ & $0.25 \pm 0.14$ \\
\hline
\end{tabular}

Real-time RT-PCR method is used to screen for influenza A, H5, H7 and H9 subtypes in the samples. The positivity rate is defined as number of positive samples/ number of sample collected

LPM, Live poultry market, SD, Standard deviation

subtype was isolated in 1992, and since then, H9N2 has become the predominant subtype in poultry [16]. Live poultry trading and feeding patterns have caused H9N2 to become prevalent in different regions of China [17]. The wide distribution of $\mathrm{H} 9$ subtype provides the opportunity for avian virus reassortment.

Our results indicated that the prevalence of AIVs varied seasonally, with higher positivity rates of $\mathrm{H} 5, \mathrm{H} 7$ and H9 subtypes in late winter (December) and early spring (January) than in summer and autumn. Several studies have found that minor fluctuations in temperature, $\mathrm{pH}$ or salinity in aquatic habitats may enhance or diminish the persistence and infectivity of AIVs [18]. AIV transmission is promoted under cool and dry conditions [19].
Our results were consistent with those of current studies and showed that AIVs can survive well in winter and early spring with higher rate. In our study, LPMs and sewage were proved to be environmental risk factors of AIVs. The samples collected from LPMs displayed the highest positivity rates for the $\mathrm{H} 5, \mathrm{H} 7$ and $\mathrm{H} 9$ subtypes, which is consistent with previous studies. In China, a large number of poultry is traded through LPMs [20] which are known to be major sources of AIVs causing significant public health concerns. Poultry market closure is still an effective measure to prevent avian influenza infects humans or poultry [21]. We recommended that LPMs should be managed strictly. Some studies have reported that influenza viruses are waterborne pathogens 
Table 2 Influenza A, H5, H7 and H9 nucleic acid positivity rates of different sample types

\begin{tabular}{|c|c|c|c|c|c|}
\hline \multirow{3}{*}{$\begin{array}{l}\text { Type/subtype } \\
\text { Year }\end{array}$} & \multicolumn{5}{|l|}{ Positivity rate (\%) } \\
\hline & \multicolumn{5}{|c|}{ (Number of positive samples/Number of samples collected) } \\
\hline & Sewage & Chopping board & Feeding trough & Cage & Faeces \\
\hline \multicolumn{6}{|l|}{ Influenza A } \\
\hline 2014 & $37.31(2819 / 7556)$ & $33.49(2373 / 7085)$ & $26.15(2138 / 8175)$ & $17.81(2538 / 14252)$ & $12.92(2617 / 20259)$ \\
\hline 2015 & $39.03(3025 / 7750)$ & $33.74(3217 / 9535)$ & $28.19(2300 / 8158)$ & $19.76(3196 / 16174)$ & $15.05(3333 / 22141)$ \\
\hline 2016 & $40.17(3774 / 9394)$ & $40.25(4558 / 11324)$ & $27.64(2797 / 10121)$ & $24.01(4013 / 16711)$ & $18.79(4503 / 23962)$ \\
\hline 2017 & $31.03(4559 / 14692)$ & $27.03(4712 / 17433)$ & $22.48(3450 / 15347)$ & $18.19(4715 / 25919)$ & $12.34(4398 / 35648)$ \\
\hline 2018 & $35.37(3190 / 9020)$ & 30.99 (3074/9919) & $23.73(2613 / 11011)$ & $19.47(3122 / 16035)$ & $13.87(3237 / 23347)$ \\
\hline Mean $\pm S D$ & $36.58 \pm 3.59$ & $33.1 \pm 4.82$ & $25.64 \pm 2.46$ & $19.85 \pm 2.46$ & $14.59 \pm 2.56$ \\
\hline \multicolumn{6}{|l|}{$\mathrm{A} / \mathrm{H} 5$} \\
\hline 2014 & $14.56(1100 / 7556)$ & $7.86(2373 / 7085)$ & $7.38(2138 / 8175)$ & $2.15(2538 / 14252)$ & $1.9(385 / 20259)$ \\
\hline 2015 & $12.12(939 / 7750)$ & 7.07 (674/9535) & $6.06(494 / 8158)$ & $2.35(380 / 16,174)$ & $1.89(418 / 22,141)$ \\
\hline 2016 & $9.59(901 / 9394)$ & $9.04(1024 / 11324)$ & $4.48(453 / 10121)$ & $2.59(433 / 16711)$ & $2.18(522 / 23962)$ \\
\hline 2017 & $5.64(828 / 14692)$ & $4.37(762 / 17433)$ & $2.42(372 / 15347)$ & $1.48(383 / 25919)$ & $0.99(353 / 35648)$ \\
\hline 2018 & $9.20(830 / 9020)$ & $8.11(804 / 9919)$ & $3.54(390 / 11011)$ & $2.56(411 / 25919)$ & $1.77(413 / 23347)$ \\
\hline Mean \pm SD & $10.22 \pm 3.34$ & $7.29 \pm 1.77$ & $4.78 \pm 1.97$ & $2.23 \pm 0.45$ & $1.75 \pm 0.44$ \\
\hline \multicolumn{6}{|l|}{$\mathrm{A} / \mathrm{H} 7$} \\
\hline 2014 & $4.74(358 / 7556)$ & $6.68(473 / 7085)$ & $3.61(295 / 8175)$ & $3.96(564 / 14252)$ & $2.35(477 / 20259)$ \\
\hline 2015 & $4.25(329 / 7750)$ & $6.12(584 / 9535)$ & $3.33(272 / 8158)$ & $3.52(569 / 16174)$ & $2.81(622 / 22141)$ \\
\hline 2016 & $5.8(545 / 9394)$ & $8.9(1008 / 11324)$ & $2.93(297 / 10121)$ & $4.3(718 / 16711)$ & $3.19(765 / 23962)$ \\
\hline 2017 & $5.73(842 / 14692)$ & $6.31(1100 / 17433)$ & $4.06(624 / 15347)$ & $3.65(946 / 25919)$ & $2.59(924 / 35648)$ \\
\hline 2018 & $0.68(61 / 9020)$ & $0.44(44 / 9919)$ & $0.27(30 / 11011)$ & $0.28(45 / 16035)$ & $0.28(66 / 23347)$ \\
\hline Mean $\pm S D$ & $4.24 \pm 2.09$ & $5.69 \pm 3.13$ & $2.84 \pm 1.49$ & $3.14 \pm 1.62$ & $2.24 \pm 1.14$ \\
\hline \multicolumn{6}{|l|}{$\mathrm{A} / \mathrm{H} 9$} \\
\hline 2014 & $19.1(1443 / 7556)$ & $17.12(1213 / 7085)$ & $15.40(1259 / 8175)$ & $9.12(1300 / 14252)$ & $5.98(1212 / 20259)$ \\
\hline 2015 & $24(1860 / 7750)$ & $19.17(1828 / 9535)$ & $19.48(1589 / 8158)$ & $12.06(1951 / 16174)$ & $8.05(1782 / 22141)$ \\
\hline 2016 & 23.32 (2191/9394) & $21.3(2412 / 11324)$ & $16.47(1667 / 10121)$ & $13.44(2246 / 16711)$ & $9.32(2234 / 23962)$ \\
\hline 2017 & $18.48(2715 / 14692)$ & $14.34(2505 / 1433)$ & $13.12(2013 / 15347)$ & $11.4(2954 / 25919)$ & $6.36(2266 / 35648)$ \\
\hline 2018 & 23.16 (2089/9020) & $21.82(2164 / 9919)$ & $14.52(1599 / 11011)$ & $14.72(2360 / 16035)$ & 8.89 (2075/23347) \\
\hline Mean $\pm S D$ & $21.62 \pm 2.6$ & $18.75 \pm 3.09$ & $15.80 \pm 2.39$ & $12.14 \pm 2.12$ & $7.72 \pm 1.49$ \\
\hline
\end{tabular}

Real-time RT-PCR method is used to detect influenza A, H5, H7 and $\mathrm{H} 9$ subtypes in different sample types. The positivity rate is defined as number of positive samples/ number of sample collected

SD, Standard deviation

that have the capacity to infect a wide variety of hosts and undergo genetic reassortment [22] In our study, we investigated five kinds of samples related to poultry feeding, sale, and slaughter. The results indicated that sewage may carry a large number of AIVs and transmit the virus among poultry.

Our studies demonstrated virus subtypes diversity in environment-related poultry and wild bird. In our study, virus isolation results indicated that the $\mathrm{H} 5$ subtype AIVs continuously existed in environmental samples in China and the proportion of H5N1 subtype AIVs viruses decreased while the proportion of H5N6 subtype AIVs viruses increased dramatically during 2014-2017. In 2018, the proportion of H5N1 subtype AIVs significantly declined to $0.8 \%$. Liu et al. have reported the epidemiology and evolution characteristics of H5 AIVs subtype in poultry since 2007. The H5 subtype was almost exclusively H5N1 subtypes before 2012. Since 2014, H5N6, H5N2 and H5N8 subtypes AIVs emerged and H5N6 subtype became predominantly in China. In 2018, 78.0\% and $21.8 \%$ of all AIV isolates were identified as H5N6 and $\mathrm{H} 5 \mathrm{~N} 2$ subtypes respectively [23]. In accordance with these studies, our data also revealed that the $\mathrm{N}$ subtype of H5 shifted from N1 to N6 during 2014-2018 in environment related AIVs. Some control strategies were carried out in order to prevent the H5 spread, including mass vaccination in poultry in China. Vaccine strain of $\mathrm{H} 5$ subtype was updated every few years to combat 


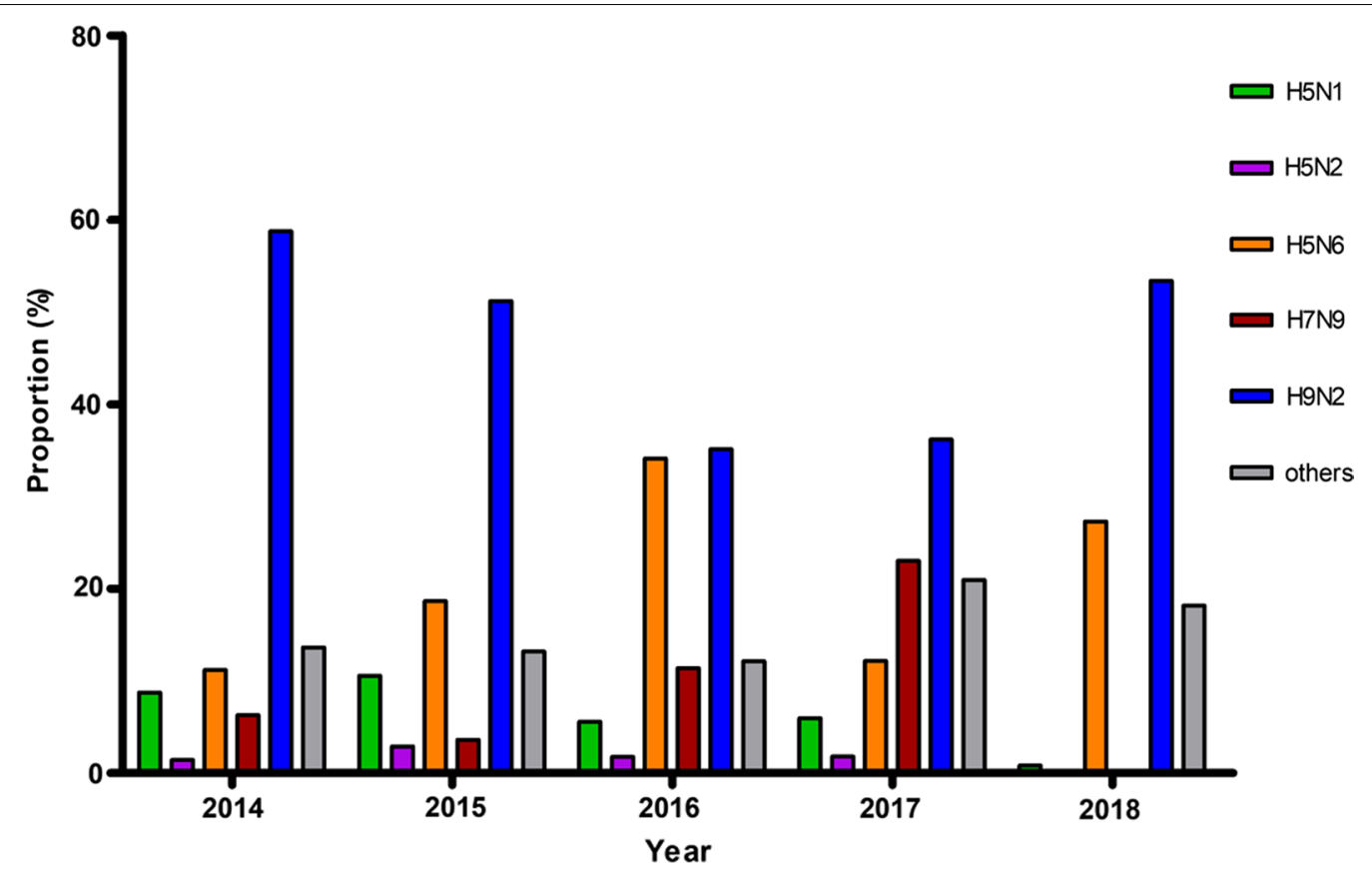

Fig. 3 Proportions of H5N1, H5N6, H7N9, H9N2 AIVs and other virus subtypes during 2014-2018. The X axis represents different years, and the Y axis represents proportion of different subtypes AIVs (\%). The proportion is defined as the number of different AlVs subtypes/number of total viruses (\%). Abbreviations Alvs: avian influenza viruses

the vaccine escape mutants. Nine vaccine strains $(\mathrm{N}-28$, Re-1,4,5,6.7.8,11,12) were used during 2005-2018 [23, 24]. The clade 7.2 of H5 AIVs were almost eliminated in chickens since 2014 largely due to mass vaccination while the clade 2.3.2 and 2.3.4 of H5 AIVs were prevalent in waterfowl and also identified in wild birds. The reason was due to the following points. Firstly, vaccination can lead to silent AIV infections and accelerate virus transmission and diversification. Second, low vaccination coverage and immune response to the vaccine reduced the vaccination effect in waterfowl [24]. Third, wild bird and waterfowl can transmit the virus each other and vaccination cannot block the transmission. Fourth, vaccine strains need to be updated accurately. These reasons may explain why H5N6 subtype AIVs substituted H5N1 subtype AIVs and became the dominant in the environment.

The avian influenza A(H7N9) virus infection was first detected in humans in March 2013. Until March 2018, a total of 1567 laboratory-confirmed human cases have been reported to WHO [25]. Some studies reported that poultry-related environments were contaminated, and poultry were infected with H7N9 AIV $s$ in the regions where H7N9 AIV s emerged [26]. Jiang et al. reported the H7N9 AIV s prevalence in poultry and evaluated the H7N9 AIV s vaccine protection efficacy in China during 2013-3018. H7N9 AIVs positivity rate varied from 2013 to 2018 in poultry, $0.25 \%$ in
$2013,0.76 \%$ in $2014,0.88 \%$ in $2015,0.41 \%$ in $2016,0.97 \%$ in 2017 and $0.00 \%$ in 2018. Large scale administration of H7-Re1vaccine made the H7N9 prevalent decrease sharply in poultry [27]. In our study, we also found that the percentage of H7N9 subtype varied in the environment, especially in 2017 (the percentage was up to 23\%) while the percentage of $\mathrm{H} 7 \mathrm{~N} 9$ decrease to $0.26 \%$ in 2018. The results indicated that the spread of the H7N9 AIV $\mathrm{s}$ in poultry and the environment has been effectively controlled and prevented human infection.

Currently, H9N2 remained highly prevalent in poultry. BJ/94-like and G1-like lineage of H9N2 AIVs have been mainly prevalent in China since the mid-1990s [28]. Since 1998, vaccination was carried out in poultry and vaccine strains were updated. However, H9N2 AIVs persisted in chickens due to antigenic drift and low vaccination coverage rate [29]. Our results indicated that H9N2 AIVs remained the highest percentage in environment samples. H9N2 AIVs prevalence in poultry and environment provided the chance for zoonotic transmission and virus reassortant.

Our study demonstrated the avian influenza virus characteristics in the environment related to poultry and wild birds in China. There were limitations in our study. Firstly, considering that there are many LPMs in China, especially in rural areas, the data in our study was collected from the environment of a small number 
of LPMs in China, there may be bias in the representativeness of the data. Secondly, the data only reflected the general features instead of individual characteristics, although the environment was related to waterfowl (duck and goose), domestic fowl (chicken) and wild bird in our study.

\section{Conclusions}

Our findings indicate that environments associated with poultry may contribute to the transmission of AIVs. The widespread and persistent circulation of avian influenza viruses in China increases the risk of zoonotic transmission and encourages the timely monitoring of changes in AIVs. Long-term control strategies and early interventions need to be developed for AIV outbreaks.

\section{Abbreviations}

AIVs: Avian influenza viruses; LPMs: Live poultry markets; PAM: Provinces, municipalities and autonomous regions; HA: Hemagglutinin; NA: Neuraminidase; SD: Standard deviation.

\section{Supplementary Information}

The online version contains supplementary material available at https://doi. org/10.1186/s40249-021-00850-3.

Additional file 1: Table S1. Primer and probe sequences used in the detection of influenza virus. Table $\mathbf{S 2}$. The average nucleic acid positivity rate of influenza $\mathrm{A}, \mathrm{H} 5, \mathrm{H} 7$ and $\mathrm{H} 9$ in different provinces, municipalities and autonomous regions. Table S2-a, S2-b, S2-c, S2-d indicated the average nucleic acid positivity rate of influenza $\mathrm{A}, \mathrm{H} 5, \mathrm{H} 7$ and $\mathrm{H} 9$ respectively. Table S3. The monthly nucleic acid positivity rate of influenza $\mathrm{A}, \mathrm{H5}, \mathrm{H7}$ and $\mathrm{H} 9$ in environmental samples related poultry during 2014-2018. Table S4. Proportion of virus subtypes isolated from environmental samples during 2014-2018.

\section{Acknowledgements}

Not applicable.

\section{Authors' contributions}

Writing: review and editing: DW. Funding acquisition: DW. Supervision: DW; YS Conceptualization: DW. Methodology: HB, YZ, LD, JD, XL, XZ, ZL. Writing: original draft preparation: HB. All authors read and approved the final manuscript.

\section{Funding}

The study was funded by National Science and Technology Major Project (2018ZX10711-001; 2020ZX10001-016; 2018ZX10734404); National Key Research and Development Program of China (2016YFD0500208) and Emergency Prevention and Control Project of Ministry of Science and Technology (10600100000015001206).

\section{Availability of data and materials}

The datasets used and analysed during the current study are available from the corresponding author on reasonable request.

\section{Declarations}

Ethics approval and consent to participate Not applicable.

\section{Consent for publication}

Not applicable.

\section{Competing interests}

The authors declared that they have no conflicts of interest.

\section{Author details}

${ }^{1}$ National Institute for Viral Disease Control and Prevention, Chinese Center for Disease Control and Prevention; WHO Collaborating Center for Reference and Research On Influenza; Key Laboratory for Biosafety, National Health Commission, Beijing, China. ${ }^{2}$ Public Health School (Shenzhen), Sun Yat-Sen University, Guangzhou, China.

Received: 28 January 2021 Accepted: 21 April 2021

Published online: 06 May 2021

\section{References}

1. Perroncito E. Epizooziatifoideneigallinacei. Annali Accad Agric. 1878;21:87

2. Burnet FM, Ferry JD. Thedifferetiation of fowl plague and Newcastle disease: experiments using the technique of chorio-allantoic membrane inoculation of the developing egg. Br J ExperimPathol. 1934;15:56.

3. Peiris JS, de Jong MD, Guan Y. Avian influenza virus (H5N1): a threat to human health. Clin Microbiol Rev. 2007;20(2):243-67.

4. Kim JK, Negovetich NJ, Forrest HL, Webster RG. Ducks: the "Trojan horses" of H5N1 influenza. Influenza Other Respir Viruses. 2009;3(4):121-8.

5. Li YT, Linster M, Mendenhall IH, Su YCF, Smith GJD. Avian influenza viruse in humans: lessons from past outbreaks. Br Med Bull. 2019;132(1):81-95.

6. WHO Group. Emergencies preparedness, response. WHO. 2021 https:// www.who.int/csr/don/26-feb-2021-influenza-a-russian-federation/en/. Accessed 26 Feb 2021.

7. Zhou J, Wu J, Zeng X, Huang G, Zou L, Song Y, et al. Isolation of H5N6, H7N9 and H9N2 avian influenza A viruses from air sampled at live poultry markets in China, 2014 and 2015. Euro Surveill. 2016:21(35):30331-4.

8. Shi J, Deng G, Ma S, Zeng X, Yin X, Li M, et al. Rapid evolution of H7N9 highly pathogenic viruses that emerged in China in 2017. Cell Host Microbe. 2018;24(4):558-68.

9. Zhou X, Li Y, Wang Y, Edwards J, Guo F, Clements AC, et al. The role of live poultry movement and live bird market biosecurity in the epidemiology of influenza A (H7N9): a cross-sectional observational study in four eastern China provinces. J Infect. 2015;71(4):470.

10. Yuan J, Lau EH, Li K, Leung YH, Yang Z, Xie C, et al. Effect of live poultry market closure on avian influenza A(H7N9) virus activity in Guangzhou, China, 2014. Emerg Infect Dis. 2015;21(10):1784-93.

11. Liao Q, Lam WT, Leung GM, Jiang C, Fielding R. Live poultry exposure, Guangzhou, China, 2006. Epidemics. 2009;1(4):207-12.

12. Zhang H, Li Y, Chen J, Chen Q, Chen Z. Perpetuation of H5N1 and H9N2 avian influenza viruses in natural water bodies. J Gen Virol. 2014;95(Pt 7):1430-5.

13. WHO Group. WHO Manual for the laboratory diagnosis and virological surveillance of influenza. 2011 https://apps.who.int/iris/handle/10665/ 44518. Accessed Jan 2011.

14. Ma MJ, Yang Y, Fang LQ. Highly pathogenic avian H7N9 influenza viruses: recent challenges. Trends Microbiol. 2019;27(2):93-5.

15. Bi Y, Chen Q, Wang Q, Chen J, Jin T, Wong G, et al. Genesis, evolution and prevalence of H5N6 avian influenza viruses in China. Cell Host Microbe. 2016;20(6):810-21.

16. Chen B, Zhang Z, Chen W. The study of avian influenza: I. The isolation and preliminary serological identification of avian influenza virus in chicken. Chin JVet Med. 1994:20:3-5.

17. Gu M, Xu L, Wang X, Liu X. Current situation of H9N2 subtype avian influenza in China. Vet Res. 2017;48(1):49.

18. Stallknecht DE, Kearney MT, Shane SM, Zwank PJ. Effects of pH, temperature, and salinity on persistence of avian influenza viruses in water. Avian Dis. 1990;34(2):412-8

19. Brown JD, Swayne DE, Cooper RJ, Burns RE, Stallknecht DE. Persistence of H5 and H7 avian influenza viruses in water. Avian Dis. 2007;51(1 Suppl):285-9. 
20. Su S, Bi Y, Wong G, Gray GC, Gao GF, Li S. Epidemiology, evolution, and recent outbreaks of avian influenza virus in China. J Virol. 2015;89(17):8671-6.

21. Yu H, Wu JT, Cowling BJ, Liao Q, Fang VJ, Zhou S, et al. Effect of closure of live poultry markets on poultry-to-person transmission of avian influenza A H7N9 virus: an ecological study. Lancet. 2014;383(9916):541-8.

22. Sooryanarain $\mathrm{H}$, Elankumaran $\mathrm{S}$. Environmental role in influenza virus outbreaks. Annu Rev AnimBiosci. 2015;3:347-73.

23. Liu S, Zhang Q, Wang S, Jiang W, Jin J, Peng C, et al. Control of avian influenza in China: strategies and lessons. TransboundEmerg Dis. 2020;67(4):1463-71.

24 Chen H. H5N1 avian influenza in China. Sci China C life Sci. 2009;52(5):419-27.

25. WHO Group. Influenza summary and assessment. WHO. 2018. https:// www.who.int/influenza/human_animal_interface/Influenza_Summary_ IRA_HA_interface_02_03_2018.pdf?ua=1. Accessed 3 March 2018.
26. He F Chen EF, LiFD, Wang XY Wang XX, Lin JF. Human infection and environmental contamination with Avian Influenza A (H7N9) Virus in Zhejiang Province, China: risk trend across the three waves of infection. BMC Public Health. 2015;15:931.

27. Jiang W, Hou G, Li J, Peng C, Wang S, Liu S, et al. Prevalence of H7N9 subtype avian influenza viruses in poultry in China, 2013-2018. TransboundEmerg Dis. 2019;66(4):1758-61.

28. Sun Y, Pu J, Jiang Z, Guan T, Xia Y, Xu Q, et al. Genotypic evolution and antigenic drift of H9N2 influenza viruses in China from 1994 to 2008. Vet Microbiol. 2010;146:215-25.

29. Zhang P, Tang Y, Liu X, Peng D, Liu W, Liu H, et al. Characterization of H9N2 influenza viruses isolated from vaccinated flocks in an integrated broiler chicken operation in eastern China during a 5 year period (1998-2002). J Gen Virol. 2008:89:3102-12.
Ready to submit your research? Choose BMC and benefit from:

- fast, convenient online submission

- thorough peer review by experienced researchers in your field

- rapid publication on acceptance

- support for research data, including large and complex data types

- gold Open Access which fosters wider collaboration and increased citations

- maximum visibility for your research: over $100 \mathrm{M}$ website views per year

At BMC, research is always in progress.

Learn more biomedcentral.com/submissions 